\title{
FAVARD CLASSES FOR $n$-DIMENSIONAL SINGULAR INTEGRALS
}

\author{
BY P. L. BUTZER AND R. J. NESSEL ${ }^{1}$
}

Communicated by E. Hewitt, November 30, 1965

Let $f \in L_{p}\left(E^{n}\right), 1 \leqq p<\infty, E^{n}$ being the $n$-dimensional Euclidean space, and let us consider an approximation process defined by means of a singular integral of Fourier convolution type

$$
K(f ; x ; \zeta)=\frac{1}{(2 \pi)^{n / 2}} \int_{E^{n}} f(x-u) k(u ; \zeta) d u .
$$

Here $u, x$ denote vectors of $E^{n}$ and $\zeta$ a positive parameter, whereas $k(u ; \zeta)$ is said to be the kernel of the integral (1) subject to the following conditions $[1$, p. 1$]$ :

$$
\begin{aligned}
\|k(\cdot ; \zeta)\|_{1} \leqq M, \quad \int_{E^{n}} k(u ; \zeta) d u=(2 \pi)^{n / 2} & \text { for all } \zeta>0 \\
\lim _{\zeta \rightarrow \infty} \int_{|u| \geq \delta}|k(u ; \zeta)| d u=0 & \text { for all } \delta>0 .
\end{aligned}
$$

It is well known $[1$, p. 10] that under these conditions the singular integral (1) exists a.e., again belongs to $L_{p}\left(E^{n}\right)$ and satisfies the relations $\|K(f ; \cdot ; \zeta)\|_{p} \leqq\|k(\cdot ; \zeta)\|_{1}\|f(\cdot)\|_{p}$ and

$$
\lim _{\zeta \rightarrow \infty}\|K(f ; \cdot ; \zeta)-f(\cdot)\|_{p}=0
$$

Starting with relation (2) one wishes to establish some connections between the rapidity of the convergence in (2) and further properties of the function $f$. Here we only aim to discuss a special but nevertheless important case of this general approximation problem, namely the case of the best possible rate of approximation of nontrivial functions $f$ by the singular integral (1), and to determine the exact class $F$ of functions $f$ for which this optimal rate is precisely attained. This notion, the so-called saturation of the process (1), was first introduced by J. Favard [6] and is, for our situation, given by

DEFINITION. Let the singular integral (1) be given with kernel $k(x ; \zeta)$ and $f \in L_{p}\left(E^{n}\right), 1 \leqq p<\infty$. If there exists a monotone decreasing

1 The results of this paper were announced by R. J. Nessel in talks held on September 15, 1964 at the Austrian Mathematical Congress, Graz, and on March 6, 1964 and August 5, 1965 at the Mathematical Research Institute, Oberwolfach, Black Forest. 
function $\phi(\zeta)$ with $\lim _{\zeta \rightarrow \infty} \phi(\zeta)=0$ and a class $F \subset L_{p}\left(E^{n}\right)$ such that

(a) $\|K(f ; \cdot ; \zeta)-f(\cdot)\|_{p}=o(\phi(\zeta))$ as $\zeta \rightarrow \infty$ implies $f(x)=0$ a.e.;

(b) $\|K(f ; \cdot ; \zeta)-f(\cdot)\|_{p}=O(\phi(\zeta))$ as $\zeta \rightarrow \infty \quad$ if and only if $f \in F$,

then we call $\phi$ the order and $F$ the saturation or Favard class of the process (1). Thereby we assume that $F$ contains at least one function different from the null-function.

In discussing the saturation of the singular integral (1) further conditions must be known for the kernel. In following the welldeveloped one-dimensional theory [3], [8] it turns out that the integral transform method, first introduced by one of the authors in e.g. [3], [4], is also an appropriate tool in the $n$-dimensional situation. This method works in a very similar fashion to that, with which certain initial or boundary value problems of differential equations may be solved by using e.g. the Laplace transform. If for $1 \leqq p \leqq 2$ one applies the $n$-dimensional Fourier transform to (1) one obtains, by the convolution theorem, a separation of the kernel and the particular function $f$. So it seems to be reasonable to postulate further conditions upon the Fourier transform of the kernel. For this purpose, let $\Psi(v)$ be a function, defined and continuous in $E^{n}$ with isolated zeros such that for all $v$

$$
\lim _{\zeta \rightarrow \infty} \frac{k^{\wedge}(v ; \zeta)-1}{\phi(\zeta)}=\psi(v),
$$

where $\phi$ is defined as in the above definition and [2]

$$
h^{\wedge}(v)=\frac{1}{(2 \pi)^{n / 2}} \int_{E^{n}} e^{-i\langle v, x\rangle} h(x) d x
$$

denotes the Fourier transform of $h \in L_{1}\left(E^{n}\right),\langle v, x\rangle \equiv v_{1} x_{1}+\cdots$ $+v_{n} x_{n}$ being the inner product of the vectors $v ; x \in E^{n}$. Furthermore, suppose there exists a family $\left\{\nu_{\xi}\right\}$ of uniformly bounded measures such that the representation

$$
\frac{k^{\wedge}(v ; \zeta)-1}{\phi(\zeta)}=\psi(v) \nu_{\zeta}^{2}(v)
$$

holds for every $v \in E^{n}$ and $\zeta>0$, where $\mu^{\iota}$, analogously to (4), designates the Fourier-Stieltjes transform of the bounded measure $\mu$. Defining the Fourier transform of $f \in L_{p}\left(E^{n}\right), 1<p \leqq 2$, in the usual way ([2], [9]) we may state the first result of this note: 
THEOREM 1. Let $f \in L_{p}\left(E^{n}\right), 1 \leqq p \leqq 2$, and the kernel $k(x ; \zeta)$ of (1) satisfy (3).

(a) If there exists a function $g \in L_{p}\left(E^{n}\right)$ such that

$$
\lim _{\zeta \rightarrow \infty}\left\|\frac{1}{\phi(\zeta)}\{K(f ; \cdot ; \zeta)-f(\cdot)\}-g(\cdot)\right\|_{p}=0
$$

then $\psi(v) f^{\wedge}(v)=g^{\wedge}(v)$ (a.e. in case $\left.1<p \leqq 2\right)$. In particular, $\|K(f ; \cdot ; \zeta)-f(\cdot)\|_{p}=o(\phi(\zeta))$ as $\zeta \rightarrow \infty$ implies $f(x)=0$ a.e.

(b) If

$$
\|K(f ; \cdot ; \zeta)-f(\cdot)\|_{p}=O(\phi(\zeta)) \quad(\zeta \rightarrow \infty),
$$

then for $p=1$ there exists $a$ bounded measure $\mu$ and for $1<p \leqq 2 a$ function $g \in L_{p}\left(E^{n}\right)$ such that

$$
\psi(v) f^{\wedge}(v)=\mu^{\nu}(v) \text { for all } v \in E^{n} ; \quad \psi(v) f^{\wedge}(v)=g^{\wedge}(v) \text { a.e. }
$$

(c) If the kernel $k(x ; \zeta)$ in addition satisfies (5) (in case $1<p \leqq 2$ we suppose that the $\nu_{5}$ are absolutely continuous) then the representation (7) implies the approximation (6).

THEOREM 2. Let $f \in L_{p}\left(E^{n}\right), 1 \leqq p \leqq 2$, and the kernel $k(x ; \zeta)$ of (1) satisfy (3). Then two equivalent characterizations of the Favard classes corresponding to a saturation order $\phi(\zeta)$ and precisely expressed by (7), are given by:

(i) $\left\|\frac{1}{(2 \pi)^{n / 2}} \int_{-R}^{R} \cdots \int_{-R}^{R}\left\{\prod_{j=1}^{n}\left(1-\frac{\left|v_{j}\right|}{R}\right)\right\} e^{i\langle\cdot, v\rangle} \psi(v) f^{\wedge}(v) d v\right\|_{p}$

$$
=O(1) \quad(R \rightarrow \infty) .
$$

(ii) The functions $\{(1 / \phi(\zeta))[K(f ; x ; \zeta)-f(x)]\}$ converge weakly* as $\xi \rightarrow \infty$, i.e. in case $p=1$, there exists a bounded measure $\mu$ such that

$$
\lim _{\xi \rightarrow \infty} \int_{E^{n}}(1 / \phi(\xi))[K(f ; u ; \xi)-f(u)] h(u) d u=\int_{E^{n}} h(u) d \mu
$$

for all continuous $h$ vanishing at infinity.

Note that condition (i) is Cramer's criterion for the representation of a function as a Fourier-(Stieltjes) integral [5]. Condition (ii) is a new result also for the one-dimensional case. All proofs and further details will be published elsewhere.

As an application we will consider the singular integral of GaußWeierstraß for $f \in L_{p}\left(E^{n}\right), 1 \leqq p \leqq 2$,

(8) $W(f ; x ; t)=(4 \pi t)^{-n / 2} \int_{E^{n}} f(x-u) \exp \left\{-u^{2} / 4 t\right\} d u\left(x \in E^{n} ; t>0\right)$ 
and its approximation to the initial value $f$ as $t \rightarrow 0+$. Setting $\zeta=\left(2 t^{1 / 2}\right)^{-1}$ and $w(u)=2^{n / 2} \exp \left\{-u^{2}\right\}$, (8) is of the form (1) with $k(u ; \zeta)=\zeta^{n} w(\zeta u)$. Regarding the condition (3), we immediately see that it is fulfilled with $\phi(\zeta)=(2 \zeta)^{-2} \equiv t$ and $\psi(v)=-v^{2}$. Instead of evaluating (5) we will here prove the direct part of the saturation theorem along the following simple lines. If the representation (7) holds with $\psi(v)=-v^{2}$ then we have, e.g., $p=1$ :

$$
\begin{aligned}
{[W(f ; \cdot ; t)-f(\cdot)]^{\wedge}(v) } & \\
\quad=\int_{0}^{t}[W(d \mu ; \cdot ; \tau)]^{\wedge}(v) d \tau & =\left[\int_{0}^{t} W(d \mu ; \cdot ; \tau) d \tau\right] \wedge(v) .
\end{aligned}
$$

Here the interchange of the order of integration is permissible by Fubini's theorem. Thus by the uniqueness theorem for Fourier transforms we obtain for every fixed $t$ and almost all $x$

$$
W(f ; x ; t)-f(x)=\int_{0}^{t} W(d \mu ; x ; \tau) d \tau,
$$

from which the approximation $\|W(f ; \cdot ; t)-f(\cdot)\|_{1}=O(t)$ immediately follows. We observe that the uniqueness theorem here takes the place of an inversion theorem, a fact which may be used to simplify the proofs of the one-dimensional case, too. Now the representation (9) may be regarded as a starting point in finding equivalent characterizations of the Favard class of (8) other than those given by Theorems 1 and 2 . Summarizing we have

Theorem 3. Suppose $f \in L_{p}\left(E^{n}\right), 1 \leqq p \leqq 2$.

(a) The approximation $\|W(f ; \cdot ; t)-f(\cdot)\|_{p}=o(t)$ as $t \rightarrow 0+$ implies $f(x)=0$ a.e.

(b) The following relations are equivalent:

(i) $\|W(f ; \cdot ; t)-f(\cdot)\|_{p}=O(t) \quad(t \rightarrow 0+)$;

(ii) for $p=1$ there exists a bounded measure $\mu$ and for $1<p \leqq 2 a$ function $g \in L_{p}\left(E^{n}\right)$ such that

$$
-v^{2} f^{\wedge}(v)=\mu^{\vee}(v) \text { for all } v \in E^{n} ; \quad-v^{2} f^{\wedge}(v)=g^{\wedge}(v) \text { a.e.; }
$$

(iii) the functions $\left\{t^{-1}[W(f ; x ; t)-f(x)]\right\}$ converge weakly* as $t \rightarrow 0+$;

(iv) for $1<p \leqq 2$ the functions $\left\{t^{-1}[W(f ; x ; t)-f(x)]\right\}$ converge strongly as $t \rightarrow 0+$; for $p=1$ the strong convergence of these functions only characterizes the subclass of the Favard class for which the measure $\mu$ in (ii) is absolutely continuous;

(v) $\|S(f ; \cdot ; r)-f(\cdot)\|_{p}=O\left(r^{2}\right)(r \rightarrow 0+)$, where 


$$
S(f ; x ; r)=\frac{\Gamma(n / 2)}{2 \pi^{n / 2}} \int_{|u|=1} f(x+r u) d u
$$

denote the spherical means of $f$.

Thus we see that for $1<p \leqq 2$ the weak* and strong convergence of the functions $\left\{t^{-1}[W(f ; x ; t)-f(x)]\right\}$ are equivalent which indeed is a new contribution for the one-dimensional theory, too. Among the other examples which may be treated in an analogous way, we will here only mention that of Cauchy-Poisson. So far our explicit proofs of the direct part of the saturation theorems in fact avoid the condition (5) which is somewhat troublesome to verify in the applications, but they depend on the special structure of the singular integrals under consideration. In order to proceed to a more general situation we study the spherical means (10) of a function $f \in L_{p}\left(E^{n}\right)$. Though they are not singular integrals of the form (1) we may nevertheless apply the same methods to prove that the saturation order of the approximation of $f$ by (10) for $1 \leqq p \leqq 2$ is given by $O\left(r^{2}\right)$ as $r \rightarrow 0+$ and that the corresponding Favard class is characterized by the function $\psi(v)=-v^{2} / 2 n$. Furthermore, for $1<p \leqq 2$, an equivalent characterization for this class is the strong convergence of the functions $\left\{r^{-2}[S(f ; x ; r)-f(x)]\right\}$ as $r \rightarrow 0+$. Using these results on spherical means one may obtain the following theorem concerning kernels of type $k(x ; \zeta)=\zeta^{n} k(\zeta x)$ (which need not necessarily satisfy (5)).

Theorem 4. Let the kernel $\zeta^{n} k(\zeta x)$ of (1) be radial, i.e. there exists a function $\kappa(r)$, defined on $0 \leqq r<\infty$, such that $k(x)=\kappa(|x|)$ a.e. Suppose that the $(n+1)$ th absolute moment of $\kappa$ exists. Then for every function $f \in L_{p}\left(E^{n}\right), 1 \leqq p \leqq 2$, which may be represented in the form (with constant $c$ )

$$
\begin{aligned}
& -c v^{2} f^{\wedge}(v)=\mu^{\nu}(v) \text { with } \mu \text { a bounded measure } \quad(p=1) \text {; } \\
& -c v^{2} f^{\wedge}(v)=g^{\wedge}(v) \text { a.e. with } g \in L_{p}\left(E^{n}\right) \quad(1<p \leqq 2) \text {, }
\end{aligned}
$$

we have the approximation

$$
\|K(f ; \cdot ; \zeta)-f(\cdot)\|_{p}=O\left(\zeta^{2}\right) \quad(\zeta \rightarrow \infty) .
$$

Furthermore, for $1<p \leqq 2$ we have the formula

where

$$
\lim _{\zeta \rightarrow \infty}\left\|\zeta^{2}[K(f ; \cdot ; \zeta)-f(\cdot)]-\frac{1}{2 n c} m_{n+1}(\kappa) g(\cdot)\right\|_{p}=0,
$$

$$
m_{n+1}(\kappa)=\frac{1}{2^{(n-2) / 2} \Gamma\left(\frac{n}{2}\right)} \int_{0}^{\infty} \kappa(r) r^{n+1} d r
$$


is a constant.

The last theorem may be announced in a more general version, nevertheless we are now in a position to treat the saturation problem of the singular integral of Bochner-Riesz

(11a) $=\frac{2^{\alpha} \Gamma(\alpha+1)}{(2 \pi)^{n / 2}} R^{n} \int_{E^{n}} f(x-u)\{R|u|\}-(n / 2+\alpha) J_{n / 2+\alpha}(R|u|) d u$,

which we may rewrite in the form

$$
B^{\alpha}(f ; x ; R)=\frac{1}{(2 \pi)^{n / 2}} \int_{|v| \leq R}\left(1-\frac{v^{2}}{R^{2}}\right)^{\alpha} e^{i\langle x, v\rangle f^{\wedge}(v) d v . ~}
$$

Here $J_{\lambda}(t)$ denotes the Bessel function of order $\lambda$. The form (11b) indicates the important role which the integral (11a) plays in the summation of multiple Fourier series [7]. An elementary calculation shows that the condition (3) is fulfilled with $\phi(R)=R^{-2}$ and $\psi(v)$ $=-\alpha v^{2}$ and that we may apply Theorem 4 in case $\alpha>(n+3) / 2$ to obtain the same theorem for (11a) as Theorem 3 for (8). Further results and applications as well as the detailed proofs of the cited theorems will appear elsewhere.

\section{REFERENCES}

1. S. Bochner, Harmonic analysis and the theory of probability, University of California Press, Los Angeles, Calif. 1955.

2. S. Bochner and K. Chandrasekharan, Fourier transforms, Princeton Univ. Press, Princeton, N. J. 1949.

3. P. L. Butzer, Fourier transform methods in the theory of approximation, Arch. Rational. Mech. Anal. 5 (1960), 390-415.

4. - Integral transform methods in the theory of approximation, On Approximation Theory, edited by P. L. Butzer and J. Korevaar, Verlag, Basel Birkhaüser, 1964, pp. 12-23.

5. H. Cramer, On the representation of a function by certain Fourier-integrals, Trans. Amer. Math. Soc. 46 (1939), 190-201.

6. J. Favard, Sur l'approximation des fonctions d'une variable reelle, Colloque d'Analyse Harmonique Publication, Vol. 15, Centre National de la Recherche Scientifique, Paris, 1949, pp. 96-110.

7. V. L. Shapiro, Fourier series in several variables, Bull. Amer. Math. Soc. 70 (1964), 48-93.

8. G. Sunouchi, On the class of saturation in the theory of approximation I, Tôhoku Math. J. 12 (1960), 339-344.

9. E. C. Titchmarsh, Introduction to the theory of Fourier integrals, Oxford Univ. Press, Oxford, 1959.

The Technical. University of Aachen, Germany 